\title{
UNIQUE BALAYAGE IN FOURIER TRANSFORMS ON COMPACT ABELIAN GROUPS
}

\author{
GEORGE S. SHAPIRO
}

\begin{abstract}
Let $K$ be a compact subset of the compact abelian group $G$ and let $\Lambda$ be a subset of the dual group $\Gamma$. Unique balayage is said to be possible for $(K, \Lambda)$ if, for every $\mu$ in $M(G)$, there is a unique $\nu$ in $M(K)$ whose Fourier transform, $\hat{\nu}$, agrees on $\Lambda$ with $\hat{\mu}$.

We prove that in order that there be any $K$ with unique balayage possible for $(K, \Lambda), \Lambda$ must belong to the coset ring of $\Gamma$. The converse of this statement is false. Some examples are given for the case where $G$ is the circle group.
\end{abstract}

1. General results on unique balayage. Let $G$ be a compact abelian group (written multiplicatively) with dual group $\Gamma$ (written additively). If $K \subset G$ is compact and $\Lambda \subset \Gamma$ then, following Beurling [2], balayage is said to be possible for $(K, \Lambda)$, if, for every measure $\mu \in M(G)$ there is a measure $\nu \in M(K)$ with

$$
\hat{\mu}(\lambda)=\hat{\nu}(\lambda) \text { for all } \lambda \in \Lambda .
$$

Here $\hat{\mu}$ denotes the (inverse) Fourier transform

$$
\hat{\mu}(\gamma)=\int_{G} \gamma(x) d \mu(x)
$$

We would like to thank Professor Colin Graham for acquainting us with the following problem of Professor S. Hartman. Determine whether there are nontrivial examples of sets $K$ and $\Lambda$ with $K \subset T$, the circle group, such that

(1) balayage is possible for $(K, \Lambda)$ and

(2) if $\mu \in M(K)$ and $\hat{\mu} \mid \Lambda=0$ then $\mu=0$.

In any $G$ and $\Gamma$, if these conditions are satisfied we shall say that unique balayage is possible for $(K, \Lambda)$.

We recall that the coset ring of $\Gamma$ is the smallest algebra of sets containing all cosets of subgroups of $\Gamma$. Our basic result is

TheOREM 1. Given $\Lambda \subset \Gamma$, in order that there be some $K \subset G$ with unique balayage possible for $(K, \Lambda)$ it is necessary that $\Lambda$ belong to the coset ring of $\Gamma$.

REMARK. We shall see in $\S 2$ that, when $\Gamma=Z$, there are some infinite $\Lambda$ in

Received by the editors September 6, 1977.

AMS (MOS) subject classifications (1970). Primary 43A25; Secondary 42A16, 42 A72.

Key words and phrases. Balayage in Fourier transforms, compact abelian groups, idempotent measures, coset ring.

(C) American Mathematical Society 1978 
the coset ring for which unique balayage is possible and others for which it is not.

Proof of Theorem 1. We first introduce some Banach spaces and reformulate the definition of unique balayage.

$B(\Lambda)$ is the space of restrictions to $\Lambda$ of Fourier transforms and is normed by

$$
\|\phi\|_{B(\Lambda)}=\inf \{\|\mu\|: \mu \in M(G) \text { and } \hat{\mu} \mid \Lambda=\phi\} .
$$

$C_{\Lambda}(G)$ is the space of continuous functions $f$ on $G$ such that $\hat{f}(-\gamma)=0$ for all $\gamma \in \Gamma \backslash \Lambda$. Here, by $\hat{f}$ we again mean the inverse transform

$$
\hat{f}(\gamma)=\int_{G} f(x) \gamma(x) d x
$$

the integral being taken with respect to normalized Haar measure. $C_{\Lambda}(G)$ is given the uniform norm. It follows from the existence of a version of Cesàro summability on $G$ (see, for example, $\left[1\right.$, p. 56]) that $C_{\Lambda}(G)$ is the uniform closure of the set of trigonometric polynomials with frequencies in $\Lambda$. The pairing of $\phi \in B(\Lambda)$ and $f \in C_{\Lambda}(G)$ given by

$$
\langle\phi, f\rangle=\int_{G} f(x) d \mu,
$$

where $\mu \in M(G)$ is any measure with $\hat{\mu} \mid \Lambda=\phi$, represents $B(\Lambda)$ as the dual space of $C_{\Lambda}(G)$. (Use the Hahn-Banach and Riesz theorems; see, for example, [4, p. 116] and note that, since $G$ is compact, $A P_{\Lambda}(G)=C_{\Lambda}(G)$.) $C(K)$ denotes the space of complex-valued continuous functions on $K$.

We define a bounded linear operator $S: C_{\Lambda}(G) \rightarrow C(K)$ by

$$
S f=f \mid K \text {. }
$$

Regarding $M(K)$ as the dual space of $C(K)$, we see that the adjoint $S^{*}$ : $M(K) \rightarrow B(\Lambda)$ is given by

$$
S^{*} \mu=\hat{\mu} \mid \Lambda \text {. }
$$

Indeed, setting $\phi=\hat{\mu} \mid \Lambda \in B(\Lambda)$, we have, for all $f \in C_{\Lambda}(G)$,

$$
\langle\phi, f\rangle=\int_{K} f(x) d \mu(x)=\langle\mu, S f\rangle
$$

so that we must have $\phi=S^{*} \mu$.

From (2) we see that unique balayage is possible for $(K, \Lambda)$ if and only if $S^{*}$ is bijective. Supposing this to be the case, it follows [3, p. 479] that $S$ is bijective and, therefore, invertible. Indeed, for $f \in C(K), S^{-1} f$ is the unique $g \in C_{\Lambda}(G)$ with $g \mid K=f$.

Define $P: C(G) \rightarrow C_{\Lambda}(G)$ by

$$
P f=S^{-1}(f \mid K) \text {. }
$$

From the above, it follows that $P f$ is the unique $g \in C_{\Lambda}(G)$ such that $g|K=f| K$. In particular, $P$ is a bounded projection of $C(G)$ onto $C_{\Lambda}(G)$. Theorem 1, therefore, follows from 
THEOREM 2. A necessary and sufficient condition for the existence of a bounded projection $P$ from $C(G)$ onto $C_{\Lambda}(G)$ is that $\Lambda$ belong to the coset ring of $\Gamma$.

Proof. This result and the argument establishing it are very similar to a result of Rosenthal [5, Theorem 3] about projections onto translationinvariant subspaces of $L^{1}(G)$. Some of the details differ, however.

Suppose, first, that such a $P$ exists. Write $f_{t}$ for the translate, $f_{t}(x)=f(x t)$, and define an operator $Q$ on $C(G)$ by

$$
(Q f)(x)=\int_{G}\left(P f_{t}\right)\left(x t^{-1}\right) d t
$$

(integral with respect to Haar measure).

Since $G$ is compact, $t \mapsto f_{t}$ is norm continuous so that the integrand above is continuous in $t$. If $\gamma \in \Gamma$ then $\gamma \in C(G)$ and $\gamma_{t}=\gamma(t) \gamma$. Thus

$$
\begin{aligned}
(Q \gamma)(x) & =\int_{G} \gamma(t)(P \gamma)\left(x t^{-1}\right) d t \\
& =\int_{G} \gamma\left(x t^{-1}\right)(P \gamma)(t) d t=\gamma(x)(P \gamma)^{\wedge}(-\gamma) .
\end{aligned}
$$

Now, $P \gamma \in C_{\Lambda}(G)$ so that, if $\gamma \notin \Lambda$ then $Q \gamma=0$. On the other hand, if $\gamma \in \Lambda$ then $P \gamma=\gamma$ so that $Q \gamma=\gamma$ also. Thus, $Q$ maps trigonometric polynomials into trigonometric polynomials belonging to $C_{\Lambda}(G)$. Since we clearly have $\|Q f\|_{\infty} \leqslant\|P\|\|f\|_{\infty}$, the fact that every function in $C(G)$ can be uniformly approximated by trigonometric polynomials now implies that, for $f \in C(G), Q f$ is continuous and, hence, that $Q f \in C_{\Lambda}(G)$. (Thus, $Q$ is the "obvious" projection of $C(G)$ onto $C_{\Lambda}(G)$.)

Now, if we regard $Q$ as an operator from $C(G)$ to itself, then $Q^{*}$ : $M(G) \rightarrow M(G)$. If $\mu \in M(G)$, then,

$$
\left(Q^{*} \mu\right)^{\wedge}(\gamma)=\left\langle\gamma, Q^{*} \mu\right\rangle=\langle Q \gamma, \mu\rangle
$$

which is $\hat{\mu}(\gamma)$ or 0 according as $\gamma \in \Lambda$ or $\gamma \notin \Lambda$.

In particular, if $\delta$ is the unit point mass at 1 , and if $\alpha=Q^{*} \delta$ then $\hat{\alpha}$ is the characteristic function of $\Lambda$ so that $\alpha$ is idempotent. By Cohen's general result on idempotent measures, [6, Theorem 3.1.3], $\Lambda$ must belong to the coset ring of $\Gamma$.

Conversely, if $\Lambda$ belongs to the coset ring of $\Gamma$, then so does $-\Lambda$ and there is a measure $\alpha \in M(G)$ such that $\hat{\alpha}$ is the characteristic function of $-\Lambda$. In this case, if we set $P f=f * \alpha$, then $P$ is a bounded projection of $C(G)$ onto $C_{\Lambda}(G)$.

2. Examples in the circle group. In general, if $\Lambda$ is finite, then unique balayage is possible for $(K, \Lambda)$ if and only if $K$ has the same cardinality as $\Lambda$ and the characters in $\Lambda$, considered as functions on $K$, are linearly independent (for then, $C_{\Lambda}(G) \mid K$ has the same dimension as $C(K)$ ).

For less trivial results, we turn to the case $G=T$, the circle group (realized as $\{z:|z|=1\})$ so that $\Gamma=Z$. We note that $\Lambda$ belongs to the coset ring of $Z$ 
if and only if $\Lambda$ differs from a periodic set by at most a finite number of elements. We present, first, a class of examples showing that the converse to Theorem 1 fails.

Let $n>1$ be an integer. Let $B$ be a nontrivial, proper subset of $\{0,1,2, \ldots, n-1\}$, containing $b$ elements. Let $n Z$ be the subgroup of multiples of $n$. Set

$$
\Lambda=\bigcup_{j \in B}(j+n Z) .
$$

THEOREM 3. With $\Lambda$ as above, there is no compact $K \subset T$ with unique balayage possible for $(K, \Lambda)$.

Proof. Let $\omega=\exp (2 \pi i / n)$ and let $\delta(z)$ denote the unit point mass at $z \in T$.

Set, for $j=0,1,2, \ldots, n-1$,

$$
\mu_{j}=\frac{1}{n} \sum_{k=0}^{n-1} \omega^{j k} \delta\left(\omega^{k}\right)
$$

Then

$$
\hat{\mu}_{j}(m)=\frac{1}{n} \sum_{k=0}^{n-1}\left(\omega^{j+m}\right)^{k}
$$

which is 1 or 0 according as $j+m \in n Z$ or not. Thus, $\hat{\mu}_{j}$ is the characteristic function of the coset $-j+n Z$. Let $D$ be the complement of $B$ in $\{0,1,2, \ldots, n-1\}$ so that $D$ has $d=n-b$ elements. In order for $f$ to belong to $C_{\Lambda}(T)$ it is, therefore, necessary and sufficient that

$$
f * \mu_{j}=0 \text { for all } j \in D .
$$

More explicitly, $f \in C_{\Lambda}(T)$ if and only if, for each $z \in T$,

$$
\sum_{k=0}^{n-1} \omega^{j k} f\left(z \omega^{-k}\right)=0 \quad \text { for all } j \in D .
$$

Let $H$ be the subgroup $\left\{1, \omega, \omega^{2}, \ldots, \omega^{n-1}\right\}$ of $T$. For each $z \in T$, (3) is a set of relations which $f \mid z H$ must satisfy. Since the $n \times n$ matrix whose $j k$ th element is $\omega^{j k}$ has orthogonal rows the $d$ relations in (3) are linearly independent. Thus, $C_{\Lambda}(T) \mid z H$ has dimension $n-d=b$. Since $C(K) \mid z H$ has as its dimension the cardinality of $K \cap z H$, in order that the operator $S$ (in equation (1)) be bijective it is, therefore, necessary that for each $z \in T$, $K \cap z H$ have exactly $b$ elements. Equivalently, each $z \in T$ must belong to exactly $b$ of the translates $\omega^{k} K, k=0,1,2, \ldots, n-1$.

No compact set $K$ can have this property. Indeed, for each $W \subset H$ which has $b$ elements, define

$$
K_{W}=\bigcap_{z \in W} z K .
$$

If $W_{1} \neq W_{2}$ then any element in $K_{W_{1}} \cap K_{W_{2}}$ would belong to at least $b+1$ of the $\omega^{k} K$. Thus, the various $K_{W}$ are disjoint. Clearly, no $K_{W}$ is all of $T$ but 
we must have

$$
T=\bigcup_{W} K_{W} .
$$

Since each $K_{W}$ is compact, this contradicts the connectedness of $T$ and so proves Theorem 3.

For some infinite sets in the coset ring of $Z$, unique balayage is possible, as the following example shows. Let $\Lambda=2 Z \cup\{1\}$ and let $K=\left\{e^{i \theta}: 0<\theta \leqslant\right.$ $\pi$ ). Note that a continuous $g$ belongs to $C_{2 Z}(T)$ if and only if $g\left(e^{i \theta}\right)$ has period $\pi$.

Suppose $f \in C(K)$. Set

$$
g(z)=f(z)+\frac{1}{2}(f(-1)-f(1)) z
$$

for $z \in K$. Then $g(1)=g(-1)$ so that $g$ may be extended to a function $h \in C_{2 Z}(T)$. Then, if

$$
f_{1}(z)=h(z)-\frac{1}{2}(f(-1)-f(1)) z
$$

we see that $f_{1} \in C_{\Lambda}(T)$ and that $f_{1} \mid K=f$ so that $S$ as defined in equation (1) is onto.

On the other hand, suppose $f \in C_{\Lambda}(T)$ and $f \mid K=0$. If

$$
h(z)=f(z)-\hat{f}(-1) z
$$

then $h \in C_{2 Z}(T)$ so that $h\left(e^{i \theta}\right)$ has period $\pi$. Thus

$$
\begin{aligned}
\hat{h}(2 m) & =\int_{0}^{\pi} h\left(e^{i \theta}\right) e^{2 i m \theta} \frac{d \theta}{\pi} \\
& =\int_{0}^{\pi} f\left(e^{i \theta}\right) e^{2 i m \theta} \frac{d \theta}{\pi}-\int_{0}^{\pi} \hat{f}(-1) e^{i(2 m+1) \theta} \frac{d \theta}{\pi}=\frac{-2 i}{\pi(2 m+1)} \hat{f}(-1)
\end{aligned}
$$

because $f \mid K=0$. If $\hat{f}(-1) \neq 0$, these are the Fourier coefficients of a discontinuous step function. Thus $\hat{f}(-1)$ and, hence, $\hat{h}$ and $\hat{f}$, must vanish. Thus, $f=0$ so that unique balayage is possible for $(K, \Lambda)$.

\section{BIBLIOGRAPHY}

1. J. Benedetto, Spectral synthesis, Academic Press, New York, 1975.

2. A. Beurling, On balayage of measures in Fourier transforms, Seminar notes, Institute for Advanced Study, Princeton, N. J., 1959-1960 (unpublished).

3. N. Dunford and J. T. Schwartz, Linear operators.I, Interscience, New York, 1958.

4. Y. Meyer, Algebraic numbers and harmonic analysis, North-Holland Math. Library, No. 2, North-Holland, Amsterdam, 1972.

5. H. P. Rosenthal, Projections onto translation-invariant subspaces of $L^{p}(G)$, Function Algebras (Proc. Internat. Sympos. on Function Algebras, Tulane University), edited by F. T. Birtel, Scott Foresman, Chicago, 1966, pp. 265-275.

6. W. Rudin, Fourier analysis on groups, Interscience, New York, 1962.

Department of Mathematics, Brooklyn College, Brooklyn, New York 11210 